\title{
Mathematical modelling of non-local spore dispersion of wind-borne pathogens causing fungal diseases
}

\author{
Mustapha El Jarroudia ${ }^{\mathrm{a}, *}$, Hasan Karjoun ${ }^{\mathrm{a}, \mathrm{b}}$, Louis Kouadio ${ }^{\mathrm{c}}$, Moussa El Jarroudi ${ }^{\mathrm{d}}$ \\ a Laboratory of Mathematics and Applications, FST Tanger, Abdelmalek Essaadi University, Tangier, Morocco \\ ${ }^{\mathrm{b}}$ International Water Research Institute, Mohammed VI Polytechnic University, Green City, Morocco \\ ${ }^{c}$ Centre for Applied Climate Sciences, University of Southern Queensland, Toowoomba, Queensland, Australia \\ ${ }^{\mathrm{d}}$ Department of Environmental Sciences and Management, University of Liege, Arlon, Belgium
}

\section{A R T I C L E I N F O}

\section{Article history:}

Received 22 October 2018

Revised 21 August 2019

Accepted 2 February 2020

\section{MSC:}

35B40

$35 \mathrm{Q} 92$

$37 \mathrm{~N} 25$

\section{Keywords:}

Plant disease

Mathematical model

Non-local dispersion

Epidemic levels

Numerical simulations

\begin{abstract}
A B S T R A C T
Theoretical description of epidemics of plant diseases is an invaluable resource for their efficient management. Here we propose a mathematical model for describing the dispersal by wind of fungal pathogens in plant populations. The dispersal of pathogen spores was modelled using a non-local diffusion equation which took into account variations in wind velocity components and contained a threshold in the convolution kernel defining the non-local diffusion term. The model was analyzed and the epidemic levels and patterns of the plant disease were derived, based upon defined assumptions of the time and space variables (i.e., represented by continuous parameters), and the host population (i.e., fixed population size). Numerical applications were then performed using reported characteristic values for wheat leaf rust, stripe rust and stem rust.
\end{abstract}

(c) 2020 Elsevier Inc. All rights reserved.

\section{Introduction}

Understanding the temporal and spatial patterns of disease spread within host populations (human, animal or plants) is crucial to develop ways of controlling such diseases and minimize their potential adverse impacts [1]. Since the beginning of the past century several studies have investigated the use of mathematical modelling in order to describe endemic and recurrent epidemics and support and guide decision-making and policies e.g., [2-5]. Pioneering works include those of Hamer [6], Ross [7], and Kermack and McKendrick [8]. The latters developed in 1927 the Susceptible-Infected-Removed (SIR) epidemiological model, which is considered as one of the key results in epidemiology [4,5,8]. The SIR model predicts depending on the transmission potential of the infection the critical fraction of susceptibles in the population that must be exceeded if an epidemic is to occur [4]. It consists in a system of three ordinary differential equations:

$$
\left\{\begin{array}{l}
S^{\prime}(t)=-\alpha S(t) I(t), \\
I^{\prime}(t)=\alpha S(t) I(t)-\theta I(t), \\
R^{\prime}(t)=\theta I(t)
\end{array}\right.
$$

where $\alpha$ is the infection rate often called the per capita disease transmission rate; and $\theta$ is the removal rate of infectives.

\footnotetext{
* Corresponding author.

E-mail addresses: m.eljarroudi@uae.ma (M. El Jarroudi), karjoun.hasan@gmail.com (H. Karjoun).
} 
The first models describing the temporal progress of a plant disease epidemic was developed by van der Plank in the 1960s [9-11]. Subsequently, various mathematical models were developed for the description of the spatial and temporal dynamics of plant disease epidemics, including models adapted from the SIR model (see Cooke et al. [12] and Madden et al. [13] for a comprehensive review).

Plant pathogens can be generally divided into two main groups: the necrotrophs which kill the host and feed on its contents, and the biotrophs which establish a long term feeding relationship with the living cells of the host [14,15]. During the stage of infection, the penetrating necrotrophic fungi release a group of fungal enzymes which disrupt the cell integrity of the host tissue, causing the cell death which thereby constitutes the food supply. Whereas biotrophic fungi form specialized physiological and morphological adaptations to the living host to ensure the supply of nutrients [16]. Biotrophic fungi also require a living host to complete their life cycle.

The dispersal of plant pathogens is fundamental to the development of plant disease epidemics in agriculture. There are several different ways by which plant pathogens can be dispersed (e.g., wind, water, insects, and impact). For many economically important crop diseases caused by fungal pathogens, the main routes of dispersion are wind-borne and rainsplash borne [17]. Spores produced on infected plants are carried by wind currents and deposited on new susceptible host plants. The initial step of establishing infection is the adhesion of the spore to the plant surface. This adhesion, which is common among all fungal species, is essential to resist further displacement by wind or water. The infection process, which depends on favorable environmental conditions (air temperature, relative humidity, rainfall, etc.), consists of several phases: spore germination, formation of appressorium and penetration hyphae, and development of infection hyphae within the host tissue [16]. For example, plant pathogens, such as rust or mildews fungi, form the haustoria which represents the hostparasite interface and is specialized in nutrient uptake [18-20]. The colonization of the plant tissue takes place during the so-called latent period. After this period, the production of spores, external to the plant, will increase to a peak level, then decrease and eventually go back to zero.

The main objective of this study was to present a mathematical model describing the dispersal by wind of pathogens for polycyclic fungal plant diseases. To the best of our knowledge, the dispersion mechanisms of wind-borne pathogens have not been yet encompassed using non-local differential equations. Indeed, dispersion processes are usually modeled through diffusion or reaction-diffusion models e.g., [21,22]. Although such diffusion models provide a good description of (locally random) short-range displacements of pathogens, they are less suitable for describing displacements over longer distances by wind. Here we considered an interaction host-pathogens model with boundary conditions where the domain of interest may represent an agricultural field. The first section of the paper describes the theoritical modelling approach. Numerical applications are then performed using three wheat rust diseases (leaf rust, stripe rust and stem rust) as case studies.

\section{Interactions host plant - pathogens and non-local dispersal of pathogens}

\subsection{Background}

We suppose that time and space variables are represented by continuous parameters and that the crop fills in a bounded connected domain $\Omega \subset \mathbb{R}^{2}$. We suppose that the host population size is fixed in the field and equals to a constant $K>0$. The density of spores at time $t$ and position $x$ is denoted by $u(x, t)$, which represents the number of pathogens per unit time and per unit area. The spore dispersal is modeled by a non-local diffusion equation which takes into account variations in wind velocity components:

$$
\frac{\partial u}{\partial t}(x, t)=\int_{\Omega} J(x-y)(u(y, t)-u(x, t)) d y+f(u)(x, t)
$$

in $\Omega$, where $J(x-y) d y$ is the probability that an individual pathogen migrates from the position $y$ to the position $x$. The integral $\int_{\Omega} J(x-y) u(y, t) d y$ is the rate at which pathogens reach the location $x$ from all other locations at time $t$. The term $\int_{\Omega} J(x-y)(u(y, t)-u(x, t)) d y$ takes into account the pathogens arriving or leaving position $x$ from other sites.

The source term $f(u)(x, t)$ may depend on $u$ and on host populations. The pathogens may not enter nor leave the domain $\Omega$. In this way the dispersion of pathogens takes place only in $\Omega$. This is the analogous of what is called homogeneous Neumann boundary conditions for partial differential equations. This is a simplified case of the general Neumann boundary problem:

$$
\frac{\partial u}{\partial t}(x, t)=\int_{\Omega} J(x-y)(u(y, t)-u(x, t)) d y+f(u)(x, t)+\int_{\mathbb{R}^{2} \backslash \Omega} J(x-y) \phi(y, t) d y
$$

where the last term takes into account the prescribed flux $\phi(y, t)$ of pathogens from outside. One can also consider the case $\Omega=\mathbb{R}^{2}$ which corresponds to the study of some epidemic expanding over a continent. The analysis of this case can be studied by using some ideas from [23].

Eq. (1) involves a non-local diffusion term $\int_{\Omega} J(x-y) u(y, t) d y$ which depends on all values of $u$ in neighborhoods of $x$. This type of diffusion operator has been introduced by Kolmogorov et al. [24] to model spatial dispersals within the genetical theory framework. It has been widely used to model diffusion processes in biological and physical problems e.g., [25-28]. In our modelling approach the vertical dimension of the crop is neglected. 


\subsection{Description of the model}

The density of healthy host individuals at time $t$ and position $x$ will be denoted by $S(x, t)$. The rate of change in the density of susceptible individuals is described by the following law of mass action $\frac{\partial S}{\partial t}(x, t)=-\alpha u(x, t) S(x, t)$, where $\alpha$ is the probability of infecting a susceptible individual, which is given as

$$
\alpha=\mu \psi
$$

where $\mu$ is the homogeneous probability per unit time for spore deposition on the crop and $\psi$ is the homogeneous probability that a spore located on a susceptible individual initiates the infection.

We consider the model (1) for the dispersion of spores in which the source term $f(u)(x, t)$ corresponding to the production of pathogens by infectious hosts at time $t$ and location $x$, is defined by

$$
f(u)(x, t)=\kappa \alpha \int_{0}^{t}(u S)(x, t-s) \beta(s) d s,
$$

where $\kappa$ is the average number of pathogen particles produced per host infected that reach the end of sporulation stage and $\beta(t)$ is the sporulation curve (see Madden et al. [13, chap 5]) defined as

$$
\beta(s)= \begin{cases}0 & \text { if } s<\tau, \\ \frac{a^{b}(s-\tau)^{b-1} e^{-a(s-\tau)}}{\Gamma(b)} & \text { if } s \geq \tau,\end{cases}
$$

where $\tau$ is the latent period (i.e., the length of time between the start of the infection process and the start of the sporulation) and $a, b$ are the shape parameters of the sporulation curve.

The term $\alpha(u S)(x, t-s)=-\frac{\partial S}{\partial t}(x, t-s)$ is the number of infected hosts arising per unit of time at position $x$, which have the infection age $s$ at time $t$.

The spore dispersal density $J$ can be computed according to the motion of biological organisms as described in Othmer et al. [29] through a stochastic process called velocity-jump process. The wind-borne spore travels with a velocity $v_{1}$ in the $x_{1}$-direction and with a velocity $v_{2}$ in the $x_{2}$-direction. We denote by $\gamma$ the scattering frequency, which is the homogeneous probability per unit time for wind changing directions. The new wind direction vector $v=\left(v_{1}, v_{2}\right)$ is randomly chosen from a distribution $q(v)$.

The spore dispersal process is governed by the equation

$$
\frac{\partial p}{\partial t}(x, t, v)=-v \cdot \nabla p-(\gamma+\mu) p+\gamma q(v) \int_{\mathbb{R}^{2}} p(x, t, z) d z,
$$

where $\mu$ is the homogeneous probability per unit time for spore deposition on the crop (introduced in (2)).

The spore dispersal density $J$ is given by

$$
J(x)=\mu \int_{0}^{+\infty} e^{-\mu t} p(x, t, v) d t
$$

$J$ is the contact density which corresponds to the density of probability of spore deposition at distance $|x|$ from the origin when released with velocity $v$.

Assuming $q$ to be homogeneously distributed on the circle of radius $v=|v|, p$ is computed using Laplace-Fourier transforms [30]. We consider throughout this paper only radial solutions $\mathrm{p}$ of problem (5). This means that the dispersal probability only depends on the distance between the initial position and targets. The spore dispersal probability density $J$ is then symmetric with $J(x)=J(|x|)$.

For some limiting cases (see for details van den Bosch et al. [30]), $J$ turns out to take the form

$$
J(x)= \begin{cases}\frac{\mu^{2}}{2 \pi v^{2}} \exp \left(-\frac{\mu}{v}|x|\right) & \text { if } \mu>\gamma, \\ \frac{\mu^{2}}{8 \pi v^{2}} K_{0}\left(\frac{\mu}{v \sqrt{2}}|x|\right) & \text { if } \mu<\gamma,\end{cases}
$$

where $K_{0}$ is the zero-order modified Bessel function of the second kind. The first distribution corresponds to the ballistic regime where the average flight duration $1 / \mu$ is small compared to the average duration of the air flow in a fixed direction $1 / \gamma$. The second distribution corresponds to the diffusion regime where the average time length during which a spore is wind-borne, $1 / \mu$, is greater than the average duration of traveling in a fixed direction $1 / \gamma$ [30]. The number $\nu / \mu$ is the average distance a spore flies before deposition. 
We summarize the equations governing our model (with initial data) as

$$
\begin{cases}\frac{\partial S}{\partial t}(x, t)=-\alpha u(x, t) S(x, t) & \text { in } \Omega \times(0, \infty), \\ \frac{\partial u}{\partial t}(x, t)=\int_{\Omega} J(x-y)(u(y, t)-u(x, t)) d y+f(u)(x, t) & \text { in } \Omega \times(0, \infty), \\ S(x, 0)=S_{0}(x) \geq 0 & \text { in } \Omega, \\ u(x, 0)=u_{0}(x) \geq 0 & \text { in } \Omega,\end{cases}
$$

where $f$ is given in (3).

\subsection{Existence and uniqueness of a solution}

In order to prove the existence and uniqueness of a solution for (8), we use Banach's fixed point Theorem. Indeed, $(8)_{2}$ can be written as

$$
\frac{\partial\left(e^{A(x) t} u\right)}{\partial t}(x, t)=\int_{\Omega} e^{A(x) t} J(x-y) u(y, t) d y+e^{A(x) t} f(u)(x, t),
$$

where $A(x)=\int_{\Omega} J(x-y) d y$.

Integrating this equality with respect to the time parameter implies

$$
u(x, t)=\int_{0}^{t} e^{A(x)(s-t)} \int_{\Omega} J(x-y) u(y, s) d y d s+e^{-A(x) t} u_{0}(x)+\int_{0}^{t} e^{A(x)(s-t)} f(u)(x, s) d s,
$$

using $(8)_{3}$. Then, solving $(8)_{1}$, we obtain

$$
S(x, t)=S_{0}(x) \exp \left(-\alpha \int_{0}^{t} u(x, s) d s\right) .
$$

Let us fix $t_{0}>0$ and consider the Banach space

$$
W_{t_{0}}=C\left(\left[0, t_{0}\right] ; L^{2}(\Omega)\right),
$$

endowed with the norm $\|w\|_{W_{t_{0}}}=\max _{0 \leq t \leq t_{0}}\left\|w_{t}\right\|_{L^{2}(\Omega)}$. The set

$$
W_{t_{0}}^{+, M}=\left\{w \in W_{t_{0}} \mid 0 \leq w \leq M \text { in } \Omega \times(0,+\infty)\right\},
$$

is closed in $W_{t_{0}}$, for every $M>0$. For every function $w_{0} \in L^{2}(\Omega)$ satisfying $0 \leq w_{0} \leq M$ in $\Omega$, we define the nonlinear mapping $T_{w_{0}}$ on $W_{t_{0}}$ through

$$
T_{w_{0}}(w)(x, t)=\int_{0}^{t} e^{A(x)(s-t)} \int_{\Omega} J(x-y) w(y, s) d y d s+e^{-A(x) t} w_{0}(x)+\int_{0}^{t} e^{A(x)(s-t)} f(w)(x, s) d s .
$$

We have the following Lemma.

Lemma 1. Let $w_{0}$ and $z_{0}$ be functions of $L^{2}(\Omega)$ satisfying $0 \leq w_{0}, z_{0} \leq M$ in $\Omega$. Then, for every $w, z \in W_{t_{0}}^{+, M}$, we have

$$
\left\|T_{w_{0}}(w)-T_{z_{0}}(z)\right\|_{W_{t_{0}}}^{2} \leq C\left(t_{0}, M\right)\|w-z\|_{W_{t_{0}}}^{2}+3\left\|w_{0}-z_{0}\right\|_{L^{2}(\Omega)}^{2},
$$

where $C\left(t_{0}, M\right)=\frac{3}{2}\left(t_{0}\right)^{2}\left(\left(\kappa \alpha \max _{x \in \Omega} S_{0}(x)\left(1+2 t_{0} \alpha M\right)\right)^{2}+1\right)$.

Proof. We have

$$
\begin{aligned}
\left(T_{w_{0}}(w)-T_{z_{0}}(z)\right)(x, t)= & \int_{0}^{t} e^{A(x)(s-t)} \int_{\Omega} J(x-y)(w-z)(y, s) d y d s \\
& +\int_{0}^{t} e^{A(x)(s-t)}(f(w)-f(z))(x, s) d s+e^{-A(x) t}\left(w_{0}-z_{0}\right)(x) .
\end{aligned}
$$

Using (3) and (9), we then observe that

$$
(f(w)-f(z))(x, s)=\kappa \alpha S_{0}(x) \int_{0}^{s} \beta(s-\zeta) \times\left(\begin{array}{l}
w(x, \zeta) \exp \left(-\alpha \int_{0}^{\zeta} w(x, \xi) d \xi\right) \\
-z(x, \zeta) \exp \left(-\alpha \int_{0}^{\zeta} z(x, \xi) d \xi\right)
\end{array}\right) d \zeta .
$$

Let us define

$$
\phi(r)=(r w(x, \zeta)+(1-r) z(x, \zeta)) \times \exp \left(-\alpha \int_{0}^{\zeta}(r w(x, \xi)+(1-r) z(x, \xi)) d \xi\right) .
$$


Then, for some $r_{0} \in(0,1)$, we have

$$
\begin{aligned}
(f(w)-f(z))(x, s) & =\kappa \alpha S_{0}(x) \int_{0}^{s} \beta(s-\zeta)(\phi(1)-\phi(0)) d \zeta \\
& =\kappa \alpha S_{0}(x) \int_{0}^{s} \beta(s-\zeta) \phi^{\prime}\left(r_{0}\right) d \zeta \\
& =\kappa \alpha S_{0}(x) \int_{0}^{s} \beta(s-\zeta) \\
& \times\left(\begin{array}{l}
\times \exp \left(-\alpha \int_{0}^{\zeta}\left(r_{0} w(x, \xi)+\left(1-r_{0}\right) z(x, \xi)\right) d \xi\right) \\
\left.\times\left(\begin{array}{l}
(w, \zeta)-z(x, \zeta)) \\
-\alpha\left(r_{0} w(x, \zeta)+\left(1-r_{0}\right) z(x, \zeta)\right) \int_{0}^{\zeta}(w(x, \xi)-z(x, \xi)) d \xi
\end{array}\right)\right) d \zeta
\end{array}\right.
\end{aligned}
$$

This implies that

$$
\begin{aligned}
|(f(w)-f(z))(x, s)| & \leq \kappa \alpha S_{0}(x) \int_{0}^{s} \beta(s-\zeta) \times\left(|w-z|(x, \zeta)+\alpha(w(x, \zeta)+z(x, \zeta)) \int_{0}^{\zeta}|w-z|(x, \xi) d \xi 1\right) d \zeta \\
& \leq \kappa \alpha S_{0}(x)\left(1+2 t_{0} \alpha M\right) \int_{0}^{s} \beta(s-\zeta)|w-z|(x, \zeta) d \zeta
\end{aligned}
$$

Using Cauchy-Schwarz inequality in (10), we have

$$
\begin{gathered}
\left|T_{w_{0}}(w)-T_{z_{0}}(z)\right|^{2}(x, t) \leq \frac{3}{2} t_{0}^{2} \int_{\Omega} J^{2}(x-y) d y \int_{0}^{t} \int_{\Omega}(w-z)^{2}(y, s) d y d s+3\left(w_{0}-z_{0}\right)^{2}(x) \\
+\frac{3}{2} t_{0}^{2}\left(\kappa \alpha S_{0}(x)\right)^{2}\left(1+2 t_{0} \alpha M\right)^{2} \int_{0}^{t}(w-z)^{2}(x, s) d s,
\end{gathered}
$$

which implies that

$$
\begin{aligned}
\int_{\Omega}\left|T_{w_{0}}(w)-T_{z_{0}}(z)\right|^{2}(x, t) d x \leq \frac{3}{2}\left(t_{0}\right)^{2} \max _{0 \leq t \leq t_{0}} \int_{\Omega}(w-z)^{2}(x, t) d x+3 \int_{\Omega}\left(w_{0}-z_{0}\right)^{2}(x) d x \\
+\frac{3}{2}\left(t_{0}\right)^{2}\left(\kappa \alpha \max _{x \in \Omega} S_{0}(x)\left(1+2 t_{0} \alpha M\right)\right)^{2} \max _{0 \leq t \leq t_{0}} \int_{\Omega}(w-z)^{2}(x, t) d x
\end{aligned}
$$

from which we deduce

$$
\left\|T_{w_{0}}(w)-T_{z_{0}}(z)\right\|_{W_{t_{0}}}^{2} \leq \frac{3}{2}\left(t_{0}\right)^{2}\left(\left(\kappa \alpha \max _{x \in \Omega} S_{0}(x)\left(1+2 t_{0} \alpha M\right)\right)^{2}+1\right) \times\|w-z\|_{W_{t_{0}}}^{2}+3\left\|w_{0}-z_{0}\right\|_{L^{2}(\Omega)}^{2}
$$

which implies the estimate of the Lemma.

Theorem 2. For every non negative $u_{0} \in L^{2}(\Omega)$ and every non negative function $S_{0}$ defined on $\Omega$, there exists a unique solution $(u, S)$ to (8) such that:

1. $u \in C\left([0,+\infty) ; L^{2}(\Omega)\right), 0 \leq u \leq M$ and $S$ is given through (9).

2. u satisfies

$$
\begin{aligned}
\int_{\Omega} \frac{\partial u}{\partial t}(x, t) d x & =\int_{\Omega} f(u)(x, t) d x \\
\int_{\Omega} u(x, t) d x & =\int_{\Omega} u_{0}(x) d x+\int_{\Omega} \int_{0}^{t} f(u)(x, s) d s d x .
\end{aligned}
$$

Proof. 1. Taking $w_{0}=u_{0}, w=u$ and $z_{0}=z=0$ in Lemma 1 , we obtain

$$
\left\|T_{u_{0}}(u)\right\|_{W_{t_{0}}}^{2} \leq C\left(t_{0}, M\right)\|u\|_{W_{t_{0}}}^{2}+3\left\|u_{0}\right\|_{L^{2}(\Omega)}^{2},
$$

hence $T_{u_{0}}(u) \in W_{t_{0}}^{+, M}$, for $t_{0}$ small enough.

Taking now $w_{0}=z_{0}=u_{0}$ in Lemma 1 , we prove that $T_{u_{0}}$ is a contraction on $W_{t_{0}}^{+, M}$ for $t_{0}$ small enough. Thus, there exists a unique fixed point $u \in W_{t_{0}}^{+, M}$ of $T_{u_{0}}$. Using classical arguments on ordinary differential equations, we can extend $u$ to $[0,+\infty)$.

Finally, we observe that $(8)_{1}$ implies (9).

2. Integrating $(8)_{2}$ over $\Omega$, we get

$$
\int_{\Omega} \frac{\partial u}{\partial t}(x, t) d x=\int_{\Omega} \int_{\Omega} J(x-y) u(y, t) d x d y-\int_{\Omega} \int_{\Omega} J(x-y) u(x, t) d x d y+\int_{\Omega} f(u)(x, t) d x
$$

As $J$ is symmetric, the two first terms of the right-hand side cancel. This implies the equality $(11)_{1}$. The equality (11) $)_{2}$ is obtained when integrating the preceding equality with respect to the time parameter. 


\subsection{Final disease level}

The sporulation on a host infected may cease once the period of infectiousness has passed or when the plant resources are exhausted. The number of spores produced in the field, at any time, cannot exceed the number $\kappa K$, with $\kappa$ being the maximal number of spores produced per host infected that reaches the end of sporulation stage and $K$ is the host population size. Thus, we have the following result.

Theorem 3. For almost every $x \in \Omega, \lim _{t \rightarrow \infty} u(x, t)$ exists and is equal to some $u_{\infty}(x)$ with

$$
\begin{aligned}
& \qquad \int_{\Omega} u_{\infty}(x) d x=\int_{\Omega} u_{0}(x) d x+\kappa \int_{\Omega}\left(S_{0}(x)-S_{\infty}(x)\right) d x, \\
& \text { where } S_{\infty}(x)=S_{0}(x) \exp \left(-\alpha \int_{0}^{+\infty} u(x, s) d s\right) .
\end{aligned}
$$

Proof. We observe that

$$
\begin{aligned}
\int_{\Omega} \int_{0}^{t} f(u)(x, s) d s d x & =\kappa \int_{\Omega} \int_{0}^{t} \int_{0}^{s} \alpha(u S)(x, s-\zeta) \beta(\zeta) d \zeta d x d s \\
& =-\kappa \int_{\Omega} \int_{0}^{t} \int_{0}^{s} \frac{\partial S}{\partial s}(x, s-\zeta) \beta(\zeta) d \zeta d x d s \\
& =-\kappa \int_{\Omega} \int_{0}^{t} \int_{0}^{s} \frac{\partial}{\partial s}\left(\left(S(x, .)-S_{0}(x)\right) * \beta\right)(\zeta) d \zeta d s d x \\
& =-\kappa \int_{\Omega} \int_{0}^{t}\left(\left(S(x, .)-S_{0}(x)\right) * \beta\right)(s) d s d x \\
& =-\kappa \int_{\Omega} \int_{0}^{t}\left(S(x, t-s)-S_{0}(x)\right) \beta(s) d s d x
\end{aligned}
$$

Thus, according to Lebesgue's dominated convergence Theorem, we obtain from $(11)_{2}$

$$
\begin{aligned}
\lim _{t \rightarrow \infty} \int_{\Omega} u(x, t) d x & =\int_{\Omega} u_{0}(x) d x+\kappa \lim _{t \rightarrow \infty} \int_{\Omega} \int_{0}^{t}\left(S_{0}(x)-S(x, t-s)\right) \beta(s) d s d x \\
& =\int_{\Omega} u_{0}(x) d x+\kappa \int_{\Omega}\left(S_{0}(x)-S_{\infty}(x)\right) d x .
\end{aligned}
$$

\subsection{Secondary infection cycles}

We suppose that the host population size is fixed in the field and denote by $S(t)$ the homogeneous density of healthy individuals at time $t$ per unit area ( $S$ is independent of $x$ ). We denote by $u(x, t)$ the density of spores at time $t$ and position $x$. We consider a nonlocal dispersion model depending on a time parameter $\xi \geq 0$

$$
\begin{cases}\frac{\partial w}{\partial t}(x, t, \xi)=\int_{\Omega} J(x-y)(w(y, t, \xi)-w(x, t, \xi)) d y+f(u)(x, t, \xi) & \text { in } \Omega \times(0, \infty), \\ w(x, 0, \xi)=g(x, \xi) & \text { in } \Omega,\end{cases}
$$

where $w(x, t, \xi)$ represents the density of spores at time $t$ and position $x$ that result from the initial infection $g(x, \xi), J$ is the dispersal density given in (6), $g(y,$.$) is a given nonnegative function in L^{2}(\Omega)$ satisfying $\int_{0}^{+\infty} g(x, \xi) d \xi<+\infty$ and $f$ is defined by

$$
f(u)(x, t, \xi)= \begin{cases}\kappa \alpha \beta(t) S(\xi-t) u(x, \xi-t) & \text { if } t \leq \xi, \\ 0 & \text { if } t>\xi,\end{cases}
$$

where $\alpha$ is the homogeneous probability of infecting a healthy individual given in (2), $\beta$ is the sporulation curve defined in (4) and $\kappa$ is the average number of spores produced per host infected.

This model represents the dynamics of a plant disease described here by a lesion which is the amount of plant surface bearing sporulating colonies of fungal pathogens. The initial condition $g(x, \xi)$ corresponds to the number of spores at time $\xi$ and position $x$ resulting from an initial infection. Initial infection may be caused by external pathogens arriving from outside the host population or by internal pathogens surviving in the soil from previous crops. Let $h(x, t)$ be the number of spores deposited per unit of time at position $x$. Then $g(x, \xi)$ can take the form [31]

$$
g(x, \xi)=\int_{0}^{\xi} \alpha h(x, \xi-s) \beta(s) d s .
$$

The source term $f(x, t, \xi)$ represents the number of lesions arising per unit of time that at time $\xi$ have infection age $t$. The term

$$
\kappa \int_{\Omega} \int_{0}^{t} \alpha S(t-s) u(x, t-s) \beta(s) d s d x=\int_{\Omega} \int_{0}^{t} f(x, s, t) d s d x
$$


corresponds to the number of spores produced by secondary infection cycles. Secondary infections are those caused by pathogens produced by new lesions on infectious hosts in the population.

The interaction healthy-pathogens is described by the following equation

$$
\left\{\begin{array}{l}
S^{\prime}(t)=-\alpha S(t) \Lambda(t) \\
S(0)=S_{0}>0
\end{array}\right.
$$

where $\Lambda(t)=\int_{\Omega} w(x, t, t) d x$.

\subsubsection{An existence result}

Using the proof of Theorem 2, we can prove the following existence and uniqueness result.

Theorem 4. For every $\xi \geq 0$, (12) has a unique solution $w(., ., \xi) \in C\left([0,+\infty) ; L^{2}(\Omega)\right)$ which is nonnegative. Moreover

$$
\int_{\Omega} w(x, t, \xi) d x=\int_{\Omega} g(x, \xi) d x+\int_{\Omega} \int_{0}^{t} f(u)(x, s, \xi) d s d x .
$$

We deduce from (14) that

$$
\Lambda(t)=\int_{\Omega} g(x, t) d x+\kappa \int_{\Omega} \int_{0}^{t} \alpha S(t-s) u(x, t-s) \beta(s) d s d x .
$$

$\Lambda(t)$ is thus the number of spores produced by the entire population of lesions per unit time. Combining (13) and (15) we obtain the following result.

Corollary 5. The rate of change of healthy individuals is described by the following integro-differential equation

$$
\left\{\begin{array}{l}
S^{\prime}(t)=\alpha S(t)\left(\kappa \int_{0}^{t} S^{\prime}(t-s) \beta(s) d s-G(t)\right), \\
S(0)=S_{0}
\end{array}\right.
$$

where $G(t)=\int_{\Omega} g(x, t) d x$.

Eq. (16) is derived in Segarra et al. [31] and corresponds to a Kermach-McKendrick model. The novelty here is that the term $\Lambda(t)$ is obtained from a non-local dispersion age infection dependent problem.

\subsubsection{Final size of the epidemic}

The number $R_{0}$ defined through $R_{0}=\alpha S_{0} \int_{0}^{+\infty} \beta(s) d s=\alpha S_{0}$ is called the basic reproduction number [32]. It can be interpreted as the average number of secondary infection period. We denote by $S_{\infty}=\lim _{t \rightarrow \infty} S(t)$ the final number of healthy individuals and by $y_{\infty}=1-\frac{S_{\infty}}{S_{0}}$ the fraction of healthy individuals which will be infected during the epidemic. We have the following result.

Theorem 6. We have $y_{\infty}=1-\exp \left(-R_{0} y_{\infty}-\alpha \int_{0}^{\infty} G(t) d t\right)$.

Proof. From Corollary 5, we deduce

$$
\frac{S^{\prime}(t)}{S(t)}=\alpha\left(\kappa \int_{0}^{t} S^{\prime}(t-s) \beta(s) d s-G(t)\right) .
$$

Integrating with respect to $t$, we get

$$
\ln \left(\frac{S(\xi)}{S_{0}}\right)=\kappa \alpha \int_{0}^{\xi} \int_{0}^{t} S^{\prime}(t-s) \beta(s) d s d t-\alpha \int_{0}^{\xi} G(t) d t
$$

We observe that

$$
\begin{aligned}
\kappa \alpha \int_{0}^{\xi} \int_{0}^{t} S^{\prime}(t-s) \beta(s) d s d t & =\kappa \alpha \int_{0}^{\xi} \int_{0}^{t}\left(S-S_{0}\right)^{\prime}(t-s) \beta(s) d t d s \\
& =\kappa \alpha \int_{0}^{\xi}\left(\left(S-S_{0}\right) * \beta\right)(t) d t \\
& =\kappa \alpha \int_{0}^{\xi} \beta(s)\left(S(\xi-s)-S_{0}\right) d s
\end{aligned}
$$

Then, substituting in (17), we obtain

$$
\ln \left(\frac{S(\xi)}{S_{0}}\right)=\kappa \alpha \int_{0}^{\xi}\left(S(\xi-s)-S_{0}\right) \beta(s) d s-\alpha \int_{0}^{\xi} G(t) d t
$$


Table 1

Weather conditions conducive to the development of wheat rust diseases and daily sporulation of pathogens $[17,44,45]$.

\begin{tabular}{lllll}
\hline Pathogen & $\mathrm{T}_{\min }\left({ }^{\circ} \mathrm{C}\right)$ & $\mathrm{T}_{\text {opt }}\left({ }^{\circ} \mathrm{C}\right)$ & $\mathrm{T}_{\max }\left({ }^{\circ} \mathrm{C}\right)$ & Daily sporulation (spores/lesion) \\
\hline P. triticina & 2 & 15 & $<30$ & $2.10^{3}$ \\
P. striiformis & 0 & $5-14$ & 20 & $15.10^{3}$ \\
P. graminis & 4 & 23,5 & 29 & $20.10^{3}$ \\
\hline
\end{tabular}

Table 2

Latent period, infection period and infection efficiency of the pathogens causing three wheat rust diseases [17].

\begin{tabular}{lllll}
\hline Pahogen & Disease & Latent period (day) & Infection period (day) & Infection efficiency (\%) \\
\hline P. triticina & Leaf rust & $7-8$ & 20 & 40 \\
P. striiformis & Stripe rust & $10-14$ & 30 & $>40$ \\
P. graminis & Stem rust & $7-9$ & 26 & $20-45$ \\
\hline
\end{tabular}

from which we deduce, as $\xi$ tends to $\infty$

$$
\begin{aligned}
\ln \left(\frac{S_{\infty}}{S_{0}}\right) & =\kappa \alpha\left(S_{\infty}-S_{0}\right) \int_{0}^{\infty} \beta(s) d s-\alpha \int_{0}^{\infty} G(t) d t \\
& =\kappa \alpha\left(S_{\infty}-S_{0}\right)-\alpha \int_{0}^{\infty} G(t) d t \\
& =-R_{0}\left(1-\frac{S_{\infty}}{S_{0}}\right)-\alpha \int_{0}^{\infty} G(t) d t .
\end{aligned}
$$

\section{Numerical applications: Case studies of wheat rusts}

\subsection{Wheat rust diseases}

Wheat rusts [leaf rust (WLR, commonly known as brown rust), stripe rust (WSR, commonly referred to as yellow rust), and stem rust (also known as black stem rust, BSR)] are among the most damaging diseases of wheat worldwide [33-36]. Yield losses associated to wheat rusts reach up to $70 \%$ in susceptible wheat cultivars, when the diseases are uncontrolled $[33,37]$. The pathogens (Puccinia triticina Roberge ex Desmaz., P. striiformis Westend f. sp. tritici Ericks, and Puccinia graminis Pers. f. sp. tritici Erikss. \& Henn, causing WLR, WSR and BSR, respectively) of these polycyclic fungal diseases can easily spread and travel long distances by dispersal of wind-borne spores (from cetimeters to thousands of kilometers) [38,39]. Only low levels of inoculum are required for initiating an epidemic, with the ability to develop rapidly under optimal weather conditions [40-42].

Weather conditions, namely temperature affects the latent period (the time between infection and the appearance of disease symptoms) and the infectious period (the length of time between the start of spore production and the end of spore production or sporulation by infected units). For P. striiformis, the infectious period tends to be longer than for the other two fungi (Table (2)). An exception is P. triticina in which sporulation continued for up to two months under optimum conditions and low lesion density (Mehta and Zadoks [43]). This long sporulation period is interpreted as a survival mechanism.

The disease cycle speeds up at higher temperatures, resulting in a faster development of the epidemic. The favourable weather conditions conducive to the development of these three wheat rusts, as well as some characteristics of infection by their pathogens are provided in Tables 1 and 2.

\subsection{Numerical applications}

In this section we present a numerical resolution of the problem (8). For simplicity, we restrict the study to a 1D case, choosing the domain $\Omega=[0,1]$. We cover the domain $\Omega \times[0, T]$ with an $N_{x} \times N_{t}$ mesh, thus introducing the spatial step size $\delta x=1 / N_{x}$ and the time step size $\delta t=T / N_{t}$. The grid points $\left(x_{i}, t_{j}\right)$ are thus given by $x_{i}=i \delta x, i=1,2, \ldots, N_{x}+1, t_{j}=j \delta t$, $j=1,2, \ldots, N_{t}+1$.

Let $u_{i}^{j}$ (resp. $S_{i}^{j}$ ) be the approximation of $u\left(x_{i}, t_{j}\right)$ (resp. $\left.S\left(x_{i}, t_{j}\right)\right)$. The convolution product $\int_{\Omega} J(x-y)(u(y, t)-u(x, t)) d y$ is approximated as follows

$$
\left(\int_{\Omega} J(x-y)(u(y, t)-u(x, t)) d y\right)_{i}^{j} \simeq \sum_{p=1}^{N_{x}+1} J\left(x_{i}-x_{p}\right)\left(u_{p}^{j}-u_{i}^{j}\right) \delta x,
$$

for every $i=1,2, \ldots, N_{x}+1, j=1,2, \ldots, N_{t}+1$, and $J$ is computed using (7). In a similar way, the term $f\left(u\left(x_{i}, t_{j}\right)\right)$ involves a convolution product with respect to the time parameter. It can be computed as

$$
f\left(u_{i}^{j}\right) \simeq \sum_{l=1}^{j} \beta\left(t_{j}-t_{l}\right)(u S)_{i}^{l} \delta t \kappa \alpha .
$$


Table 3

Values of parameters used for the numerical application of Eq. (8).

\begin{tabular}{lllllll}
\hline Pathogen & $\kappa$ & $\alpha$ & $\frac{v}{\mu}$ & $\tau$ (days) & $b$ & $a$ \\
\hline P. triticinia & $40.10^{3}$ & 0.18 & 2.19 & 7 & 2.86 & 0.24 \\
P. striiformis & $45.10^{4}$ & 0.2 & 3.29 & 10 & 3.00 & 0.20 \\
P. graminis & $52.10^{4}$ & 0.14 & 2.19 & 7 & 3.70 & 0.21 \\
\hline
\end{tabular}
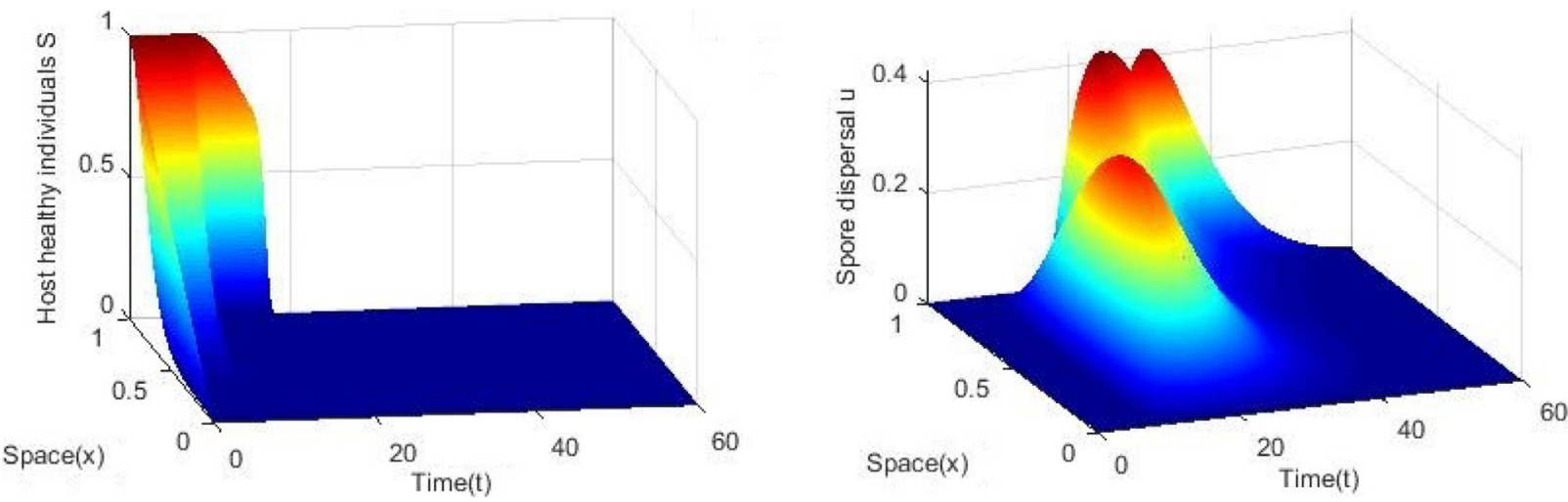

Fig. 1. Density curves of healthy host individuals $S$ (left) and spore dispersal $u$ (right) for wheat leaf rust (caused by Puccinia triticina).

The Eq. (8) $)_{1}$ is approximated using (9) and the approximation of the integral based on the trapezoidal rule

$$
S_{i}^{j} \simeq S_{0}\left(x_{i}\right) \exp \left(-\alpha \delta t\left(\frac{u_{i}^{j}+u_{i}^{1}}{2}+\sum_{l=1}^{j-1} u_{i}^{l}\right)\right) .
$$

The discretization of $(8)_{2}$ is obtained using an explicit rule, which finally leads to the discrete scheme

$$
\left\{\begin{array}{l}
u_{i}^{j+1}=u_{i}^{j}+\delta t\left(\sum_{p=1}^{N_{x}+1} J\left(x_{i}-x_{p}\right)\left(u_{p}^{j}-u_{i}^{j}\right) \delta x+f\left(u_{i}^{j}\right)\right), \\
S_{i}^{j+1}=S_{0}\left(x_{i}\right) \exp \left(-\alpha \delta t\left(\frac{u_{i}^{j+1}+u_{i}^{1}}{2}+\sum_{l=1}^{j} u_{i}^{l}\right)\right), \\
u_{i}^{1}=u_{0}\left(x_{i}\right) \geq 0 \\
S_{i}^{1}=S_{0}\left(x_{i}\right) \geq 0
\end{array}\right.
$$

for every $i=1, \ldots, N_{x}+1$ and $j=1, \ldots, N_{t}$.

The aerial dispersal of plant pathogens by wind is mainly a passive motion involving three events: liberation, transport and deposition. To liberate spores, the wind seed must be higher than a threshold $v$ which decreases with the duration of calm conditions: $v=1.0 \mathrm{~m} / \mathrm{s}$ for $P$. triticina and P. graminis, and $v=1.5 \mathrm{~m} / \mathrm{s}$ for $P$. striiformis. The threshold $v$ appears in the model through the ratio $\nu / \mu$ where $\mu$ is the median time to deposition (see [46, p. 1329, Fig. 3]). Given the values provided in Tables 1 and 2, and based on van den Bosch et al. [47] and Hau and de Vallavieille-Pope [17], the values of parameters in Eq. (8) were derived (Table 3).

All calculations were performed using the MATLAB software (version $x x$, The MathWorks Inc., Natick, MA). Figs. 1-3 present the density of healthy host individuals $S$ and density curves of spore dispersal $u$ for WLR, WSR and BSR, respectively. The same initial conditions $S_{0}(x)=1000 x$ and $u_{0}(x)=100\left(1-x^{2}\right)^{2}(x \in(0,1))$ are used for all three diseases. The curves were drawn for a period of 60 days.

The spatio-temporal patterns of a given wheat rust disease are described by the curve $S(x, t)$, which represent the susceptible healthy host plants at the position $x$ and time $t$. As the density $u(x, t)$ of pathogens increases, that of plant hosts slowly decreases. Figs. 1-3 show that the production of spores increases to a peak level beyond the latent period $\tau$; then decreases rapidly. In our numerical analyses the evolution of susceptible plant hosts is spatially quite homogeneous. The wind velocity leading to spore liberation, set as a constant, may explain such patterns. The aerial dispersal of spores across wheat fields is also influenced by other factors including the angle of the wind direction, the weight and shape of spores, and plant architecture. 

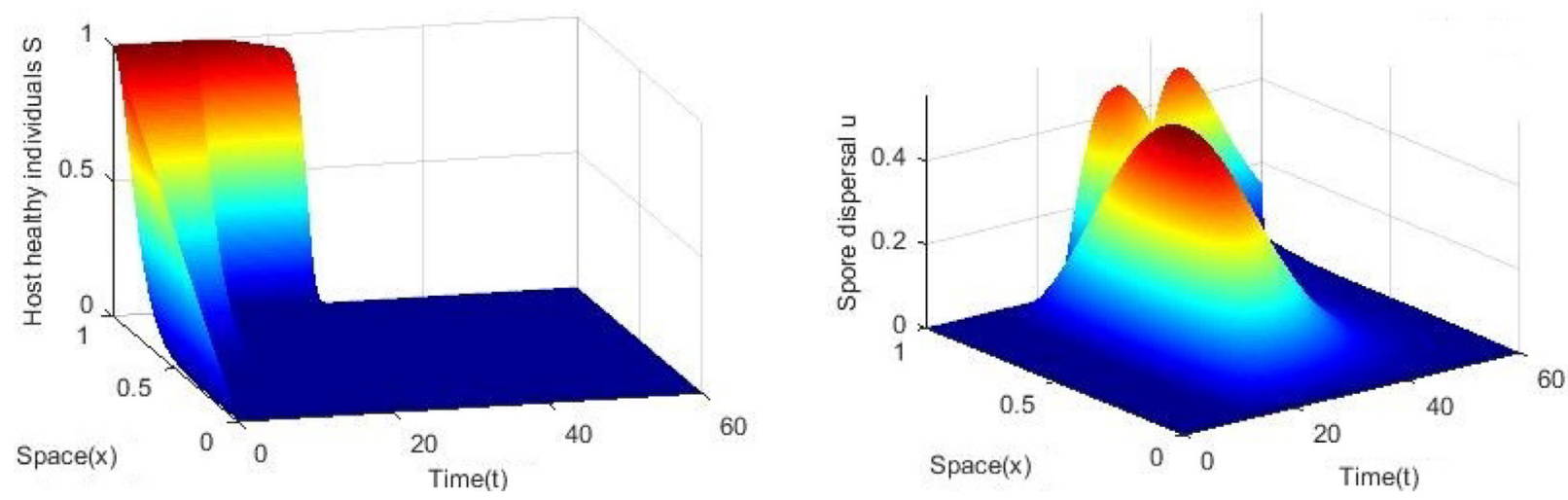

Fig. 2. Density curves of healthy host individuals $S$ (left) and spore dispersal $u$ (right) for wheat stripe rust caused by Puccinia striiformis.
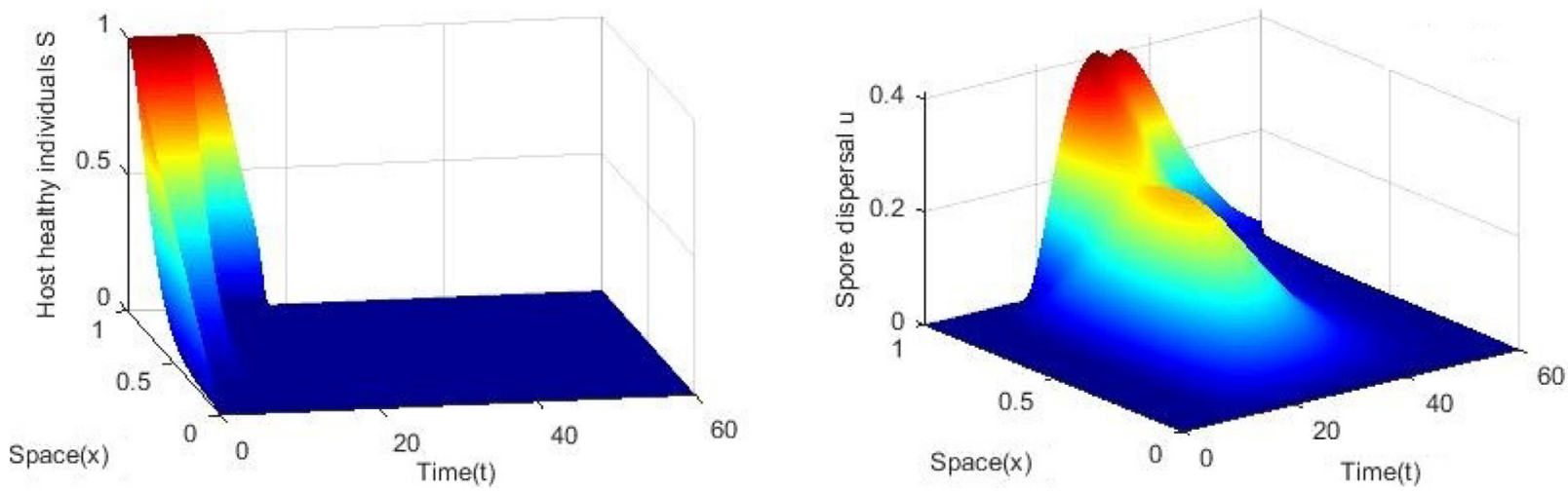

Fig. 3. Density curves of healthy host individuals $S$ (left) and spore dispersal $u$ (right) for wheat stem rust (caused by Puccinia graminis).

\section{Concluding remarks}

We proposed a mathematical model which accounts for the development of plant epidemic diseases, particularly the dispersal by wind of pathogens. The spore dispersal was modeled using a diffusion equation containing a non-local term involving a dispersal kernel. The dispersal kernel, which corresponds to the density probability function, depends on the variations of the wind velocity components. According to the non-local term, pathogens are transported in the air and deposited over new susceptible host plants where they will generate secondary foci.

Using selected parameter values retrieved from experimental data [17] the epidemic dynamics and patterns of wheat rust diseases were numerically investigated. The spatio-temporal patterns of these polycyclic diseases depend on the actual biological parameters of their pathogens, influenced by weather conditions and the susceptibility of wheat cultivars.

The probabilty of infecting a host individual, expressed by the parameter $\alpha$, depends on time varying environmental conditions such as temperature and wetness [17,40-42]. The complexity of the inter-relationships between these influencing factors make it difficult to quantify the infection probability at a given site. Nevertheless, the introduction of a time varying probability $\alpha(t)$ in our model would lead to straightforward modifications in the computations while the final results remain unchanged.

The vertical dispersal kernel of pathogens, mainly due to rain splash [48], evolves with the vertical structure (number of leaf layers) of the host individual. Such vertical dispersals was not included in our modelling approach and will be the aim of forthcoming research work. The consideration of different groups in the host population (i.e., healthy, ixnfectious and the removed or post-infectious individuals), e.g. using integro-differential equations, would help improve the present modelling approach.

\section{References}

[1] M.J. Jeger, Improved understanding of dispersal in crop pest and disease management: current status and future directions, Agric For Meteorol. 97 (4) (1999) 331-349, doi:10.1016/S0168-1923(99)00076-3.

[2] R.M. Anderson, Transmission dynamics and control of infectious disease agents, in: R.M. Anderson, R.M. May (Eds.), Population Biology of Infectious Diseases, Springer-Verlag, Berlin, Germany, 1982, pp. 149-176, doi:10.1002/9780470114209.ch22.

[3] P.E. Waggoner, Models of plant disease, BioScience 31 (4) (1981) 315-319. 
[4] M. Choisy, J.-F. Guégan, P. Rohani, Mathematical modeling of infectious diseases dynamics, in: M. Tibayrenc (Ed.), Encyclopedia of infectious diseases: Modern Methodologies, Wiley-Blackwell, Hoboken, NJ, USA, 2006, pp. 379-404, doi:10.1002/9780470114209.ch22.

[5] F. Brauer, Mathematical epidemiology: past, present, and future, Infect. Disease Model. 2 (2) (2017) 113-127, doi:10.1016/j.idm.2017.02.001.

[6] W.H. Hamer, Epidemic disease in england - the evidence of variability and of persistency of type, The Lancet 167 (4307) (1906) 733-739, doi:10.1016 S0140-6736(01)80340-8.

[7] R. Ross, The Prevention of Malaria, John Murray, London, UK, 1911.

[8] W.O. Kermack, A.G. McKendrick, A contribution to the mathematical theory of epidemics, Proc. R. Soc. Lond. A Math. Phys. Eng. Sci. 115 (772) (1927) 700-721, doi:10.1098/rspa.1927.0118.

[9] J.E. Van der Plank, Analysis of Epidemics, in: J.G. Horsfall, E.B. Cowling (Eds.), Plant Pathology: An Advance Treatise, 3, Academic Press, New York, USA, 1960, pp. 229-289.

[10] J.E. Van der Plank, Plant Diseases: Epidemics and Control, Academic Press, New York, USA, London, UK, 1963.

[11] J.E. Van der Plank, Dynamics of epidemics of plant disease, Science 147 (3654) (1965) 120-124.

[12] B.M. Cooke, J.D. Gareth, B.e. Kaye, The Epidemiology of Plant Diseases, 2, Springer Netherlands, Dordrecht, Netherlands, 2006, doi:10.1007/ 1-4020-4581-6.

[13] C.V. Madden, G. Hughes, F. van den Bosch, The study of the plant disease epidemics, The American Phytopathological Society, St. Paul, MN, USA, 2007.

[14] D. Moore, G.D. Robson, A.P.J. Trinci, 21st century guidebook to fungi, Cambridge University Press, Cambridge, 2000, doi:10.1017/CBO9780511977022.

[15] R.K. Horst, Classification of plant pathogens, in: Westcott's Plant Disease Handbook, Springer USA, Boston, MA, USA, 2001, pp. 33-63, doi:10.1007/ 978-1-4757-3376-1_2.

[16] C. Struck, Infection strategies of plant parasitic fungi, in: B.M. Cooke, J.D. Gareth, B. Kaye (Eds.), The Epidemiology of Plant Diseases, Springer Netherlands, Dordrecht, Netherlands, 2006, pp. 117-137, doi:10.1007/1-4020-4581-6_4.

[17] B. Hau, C. de Vallavieille-Pope, Wind-dispersed Diseases, in: B.M. Cooke, J.D. Gareth, B. Kaye (Eds.), The Epidemiology of Plant Diseases, Springer Netherlands, Dordrecht, Netherlands, 2006, pp. 387-416, doi:10.1007/1-4020-4581-6_15.

[18] K. Mendgen, C. Struck, R.T. Voegele, M. Hahn, Biotrophy and rust haustoria, Physiol. Mol. Plant Pathol. 56 (4) (2000) 141-145, doi:10.1006/pmpp.2000. 0264.

[19] R.C. Staples, Nutrients for a rust fungus: the role of haustoria, Trends Plant Sci. 6 (11) (2001) 496-498, doi:10.1016/S1360-1385(01)02126-4.

[20] K. Mendgen, M. Hahn, Plant infection and the establishment of fungal biotrophy, Trends Plant Sci. 7 (8) (2002) 352-356, doi:10.1016/S1360-1385(02) 02297-5.

[21] J.D. Murray, Mathematical biology, Biomathematics 19, Springer-Verlag Berlin Heidelberg, New York, USA, 1993, doi:10.1007/978-3-662-08542-4.

[22] A. Okubo, S.A. Levin, Diffusion and ecological problems: Modern perspectives, Interdisciplinary Applied Mathematics 14, Springer-Verlag New York, New York, USA, 2001, doi:10.1007/978-1-4757-4978-6.

[23] F. Andreu-Vaillo, J.J. Toledo-Melero, J.M. Mazon, J.D. Rossi, Nonlocal Diffusion Problems, 165, American Mathematical Soc., 2010.

[24] A.N. Kolmogorov, I.G. Petrovskii, N.S. Piskounov, Etude de l'équation de la diffusion avec croissance de la quantité de matière et son application à un problème biologique, Bulletin Université d'Etat de Moscou (Bjul. Moskowskogo Gos. Univ), Série internationale A1 (1937) 1-26.

[25] C. Atkinson, G.E.H. Reuter, Deterministic epidemic waves, Math. Proc. Cambridge Philos. Soc. 80 (2) (1976) 315-330, doi:10.1017/S0305004100052944.

[26] P. Bates, P.C. Fife, V. Ren, X. Wang, Travelling waves in convolution model for phase transitions, Arch Rat Mech Anal 138 (1997) $105-136$.

[27] S. Pan, W.-T. Li, G. Lin, Travelling wave fronts in nonlocal delayed reaction-diffusion systems and applications, Zeitschrift für angewandte Mathematik und Physik 60 (3) (2009) 377-392, doi:10.1007/s00033-007-7005-y.

[28] S.A. Gourley, J. Wu, Delayed nonlocal diffusion systems in biological invasion and diseases spread. nonlinear dynamics and evolution equations, volume Fields Inst Commun 48, American Mathematical Society, Providence, RI, USA, 2010.

[29] H.G. Othmer, S.R. Dunbar, W. Alt, Models of dispersal in biological systems, J. Math. Biol. 26 (3) (1988) $263-298$, doi:10.1007/BF00277392.

[30] F. van den Bosch, J.A.J. Metz, J.C. Zadoks, Pandemics of focal plant disease, a model, Phytopathology 89 (1999) 495-505, doi:10.1094/PHYTO.1999.89.6. 495.

[31] J. Segarra, M.J. Jeger, F. van den Bosch, Epidemic dynamics and patterns of plant diseases, Phytopathology 91 (2001) 1001-1010, doi:10.1094/PHYTO. 2001.91.10.1001.

[32] O. Diekmann, J.A.P. Heesterbeek, J.A.J. Metz, On the definition and the computation of the basic reproduction ratio $r_{0}$ in models for infectious diseases in heterogeneous populations, J. Math. Biol. 28 (4) (1990) 365-382, doi:10.1007/BF00178324.

[33] A.P. Roelfs, R.P. Singh, E.E. Saari, Rust diseases of wheat: Concepts and methods of disease management, CIMMYT, Mexico, D.F, Mexico, 1992.

[34] D.P. Hodson, Shifting boundaries: challenges for rust monitoring, Euphytica 179 (1) (2011) 93-104, doi:10.1007/s10681-010-0335-4

[35] M.S. Hovmøer, C.K. Sørensen, S. Walter, A.F. Justesen, Diversity of puccinia striiformis on cereals and grasses, Annu. Rev. Phytopathol. 49 (1) (2011) 197-217, doi:10.1146/annurev-phyto-072910-095230.

[36] M. EL Jarroudi, L. Kouadio, M. Beyer, J. Junk, L. Hoffmann, B. Tychon, H. Maraite, C.H. Bock, P. Delfosse, Economics of a decision-support system for managing the main fungal diseases of winter wheat in the grand-duchy of luxembourg, Field Crops Res. 172 (2015) 32-41, doi:10.1016/j.fcr.2014.11.012.

[37] E.-C. Oerke, H.-W. Dehne, Global crop production and the efficacy of crop protection - current situation and future trends, Eur. J. Plant Pathol. 103 (3) (1997) 203-215, doi:10.1023/A:1008602111248.

[38] I. sache, Short-distance dispersal of wheat rust spores by wind and rain, Agronomie 20 (2000) 757-767.

[39] D.E. Aylor, Spread of plant disease on a continental scale: role of aerial dispersal of pathogens, Ecology 84 (8) (2003) 1989-1997, doi:10.1890/01-0619.

[40] R.F. Line, Stripe rust of wheat and barley in north america: a retrospective historical review, Annu. Rev. Phytopathol. 40 (1) (2002) 75-118, doi:10. 1146/annurev.phyto.40.020102.111645.

[41] M. El Jarroudi, L. Kouadio, P. Delfosse, B. Tychon, Brown rust disease control in winter wheat: i. exploring an approach for disease progression based on night weather conditions, Environ. Sci. Pollut. Res. 21 (7) (2014) 4797-4808, doi:10.1007/s11356-013-2463-6.

[42] M. El Jarroudi, L. Kouadio, C.H. Bock, M. El Jarroudi, J. Junk, M. Pasquali, H. Maraite, P. Delfosse, A threshold-based weather model for predicting stripe rust infection in winter wheat, Plant Dis. 101 (2017) 693-703, doi:10.1094/PDIS-12-16-1766-RE.

[43] Y. Mehta, J. Zadoks, Uredospore production and sporulation period of pucciniarecondita f.sp. triticina on primary leaves of wheat., Netherlands J. Plant Pathol. 76 (1970) 267-276.

[44] M.G. Eversmeyer, C.L. Kramer, Z.M. Hassan, Environmental influences on the establishment of puccinia recondita infection structures, Plant Dis. 72 (1988) 409-412, doi:10.1094/PD-72-0409.

[45] M.G. Eversmeyer, C.L. Kramer, Epidemiology of wheat leaf and stem rust in the central great plains of the usa, Annu. Rev. Phytopathol. 38 (1) (2000) 491-513, doi:10.1146/annurev.phyto.38.1.491.

[46] A. Stockmarr, V. Andreasen, H. Østergård, Dispersal distances for airborne spores based on deposition rates and stochastic modeling, Phytopathology 97 (2007) 1325-1330, doi:10.1094/PHYTO-97-10-1325.

[47] F. van den Bosch, H.D. Frinking, J.A.J. Metz, J.C. Zadoks, Focus expansion in plant disease. iii: two experimental examples, Phytopathology 78 (1988) 919-925, doi:10.1094/Phyto-78-919.

[48] L. Huber, L. Madden, B. Fitt, Environmental biophysics applied to the dispersal of fungal spores by rain-splash, in: B.M. Cooke, J.D. Gareth, B. Kaye (Eds.), The Epidemiology of Plant Diseases, Springer Netherlands, Dordrecht, Netherlands, 2006, pp. 417-444, doi:10.1007/1-4020-4581-6_16. 